\title{
Comparison of assisted reproductive technology outcomes in infertile women with polycystic ovary syndrome: In vitro maturation, GnRH agonist, and GnRH antagonist cycles
}

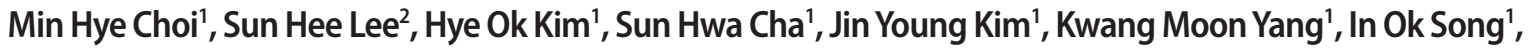 \\ Mi Kyoung Koong ', Inn Soo Kang' ', Chan Woo Park' \\ 'Division of Reproductive Endocrinology and Infertility, Department of Obstetrics and Gynecology, ${ }^{2}$ Laboratory of Reproductive Biology and Infertility, \\ Cheil General Hospital and Women's Healthcare Center, Kwandong University College of Medicine, Seoul, Korea
}

Objective: We compared the assisted reproductive technology (ART) outcomes among infertile women with polycystic ovary syndrome (PCOS) treated with IVM, conventional IVF, GnRH agonist, and GnRH antagonist cycles.

Methods: The prospective study included a total of 67 cycles in 61 infertile women with PCOS. The women with PCOS were randomized into three IVF protocols: IVM/IVF with FSH and hCG priming with immature oocyte retrieval 38 hours later (group A, 14 cycles), GnRH agonist long protocol (group B, 14 cycles), and GnRH antagonist multi-dose flexible protocol (group C, 39 cycles). IVF outcomes, such as clinical pregnancy rate (CPR), implantation rate (IR), miscarriage rate (MR), and live birth rate (LBR), were compared among the three groups.

Results: Age, BMl, and basal FSH and LH levels did not differ among the three groups. The number of retrieved oocytes and 2 pronucleus embryos was significantly lower in group A compared with groups B and C. The CPR, IR, MR, and LBR per embryo transfer showed no differences among the three groups. There was no incidence of ovarian hyperstimulation syndrome in group $A$.

Conclusion: The IR, MR, and LBR in the IVM cycles were comparable to those of the GnRH agonist and GnRH antagonist cycles. The IVM protocol, FSH and hCG priming with oocyte retrieval 38 hours later, is an effective ART option that is comparable with conventional IVF for infertile women with PCOS.

Keywords: Polycystic ovary syndrome; In vitro maturation; GnRH antagonist; GnRH agonist; Assisted reproductive technology outcome

\section{Introduction}

Polycystic ovary syndrome (PCOS) is a common endocrinopathology. The prevalence of PCOS ranges from $3 \%$ to $7 \%$ in women of re-

Received: Nov 5, 2012 · Revised: Nov 23, 2012 · Accepted: Nov 27, 2012 Corresponding author: Chan Woo Park

Department of Obstetrics and Gynecology, Cheil General Hospital and Women's Health Care Center, Kwandong University College of Medicine, 17 Seoae-ro 1-gil, Jung-gu, Seoul 100-380, Korea

Tel: +82-2-2000-7549 Fax:+82-2-2000-7790 E-mail:novak21c@yahoo.co.kr

This is an Open Access article distributed under the terms of the Creative Commons Attribution Non-Commercial License (http://creativecommons.org/licenses/by-nc/3.0/) which permits unrestricted non-commercial use, distribution, and reproduction in any medium, provided the original work is properly cited. productive age [1] and may be as high as $20 \%$ among women with infertility [2]. The diagnostic criteria for PCOS were identified at a consensus meeting and are characterized by hyperandrogenism, the presence of typical ultrasound features of polycystic ovaries, and chronic anovulation in women without specific underlying disease of the adrenal or pituitary glands [3].

For infertile women with PCOS, clomiphene citrate (CC) is the first line of therapy. Some of these women are $\mathrm{CC}$ resistant and require other treatment, such as gonadotropin, ovarian drilling, or insulin sensitizing agents [4]. A proportion of PCOS women do not respond to these conventional treatments and ultimately require assisted reproductive technology (ART). Ovarian stimulation in women with 
PCOS poses a particular challenge, as many of these women exhibit exaggerated response, resulting in an increased risk of ovarian hyperstimulation syndrome (OHSS) and multiple gestations [5]. To reduce these PCOS-related complications, a recent study recommended the use of a GnRH antagonist protocol, which resulted in a decrease in the incidence of OHSS as compared with GnRH agonists in PCOS patients [6].

As an alternative to conventional IVF, one potentially useful intervention involves immature oocyte retrieval with subsequent oocyte IVM. Since the first live birth following the collection of immature human oocytes in an unstimulated cycle [7], many successful pregnancies achieved through IVM have been reported, and over 1,000 babies have already been born [8]. A recent meta-analysis showed that IVM is a feasible option for subfertile women with PCOS [9].

The IVM technique has progressively developed over recent years, but there is still controversy surrounding IVM protocols, such as FSH priming, hCG priming, timing of oocyte retrieval, and in vitro culture conditions [10-13]. Unfortunately, there have been no randomized trials have indicated that IVM is a preferred ART option over conventional IVF for infertile women with PCOS. Furthermore, any effects of IVM relative to conventional IVF in terms of implantation and live birth rates remain unknown.

The aim of this prospective study was to compare IVM cycles using FSH and hCG priming with oocyte retrieval 38 hours later to either $\mathrm{GnRH}$ agonist or $\mathrm{GnRH}$ antagonist cycles with the primary endpoint being the implantation and live birth rate in infertile women with PCOS.

\section{Methods}

\section{Study population}

This prospective study included a total of 67 cycles of 61 infertile women with PCOS. The study participants were 28 to 41 years old and had visited Cheil General Hospital between April 2009 and November 2010. PCOS was diagnosed according to the 2003 the European Society of Human Reproduction and Embryology/the American Society for Reproductive Medicine revised criteria. According to the Rotterdam criteria, we accepted the presence of two of the three following characteristics for inclusion in the study: 1) oligomenorrhea/amenorrhea; 2) clinical findings of hyperandrogenism; and 3) polycystic ovaries on transvaginal sonography. Each participant was assigned to one of three IVF protocols: IVM/IVF with FSH and hCG priming protocol (group A, 14 cycles), GnRH agonist long protocol (group B, 14 cycles) and GnRH antagonist multi-dose flexible protocol (group C, 39 cycles). IVF outcomes, such as the OHSS, clinical pregnancy rate (CPR), clinical abortion rate (CAR), and live birth rate (LBR), were compared among the three groups.

\section{Controlled ovarian hyperstimulation}

The participants underwent IVF treatment by the IVM/IVF with FSH and hCG priming protocol (group A, 14 cycles), the GnRH agonist long protocol (group B, 14 cycles), or the GnRH antagonist multi-dose flexible protocol (group C, 39 cycles).

1) The IVM/IVF with FSH and hCG priming protocol (group A) was used in 14 cycles. On days 2 to 3 of the menstrual cycle, the pelvic cavity was evaluated by transvaginal sonogram (TVS) and blood samples for assays of $\mathrm{FSH}, \mathrm{E}_{2}$, and $\mathrm{LH}$ were obtained by venipuncture. The participants received $150 \mathrm{IU}$ of recombinant FSH (follitropin, Follitrope, LG, Iksan, Korea) administered daily starting on day 2 to 3 until day 6-8 of the menstrual cycle. TVS was repeated on cycle day 6 or 8 to monitor the leading follicle. When the follicle size was greater than $12 \mathrm{~mm}$ and the thickness of the endometrium was greater than $6 \mathrm{~mm}$, hCG (250 $\mu \mathrm{g}$ of choriogonadotropin-alfa, Ovidrel, Serono, Roma, Italy) was administered. 38 hours after hCG injection, immature oocyte retrieval was performed under TVS. The collected cumulusoocyte complexes (COCs) were assessed for the presence or absence of a germinal vesicle or the first polar body by microscope. The retrieved immature oocytes were isolated and incubated in a 4-well dish containing IVM media ( $800 \mu \mathrm{L}$ G2, VitroLife, Kungsbacka, Sweden) supplemented with $10 \%$ HSA (VitroLife), rFSH $(75 \mathrm{mlU} / \mathrm{mL}$, Gonal-F, Serono, Roma, Italy), rhCG (0.5 IU/mL, Ovidrel) and $\mathrm{E}_{2}$ (Sigma, St. Louis, MO, USA) at $37^{\circ} \mathrm{C}, 5 \% \mathrm{CO}_{2}$ in humidified air. The mature oocytes were cultured for 4 hours and immature oocytes for up to 48 hours. After culture, the oocytes were denuded by hyaluronidase solution ( $0.1 \%$ hyaluronidase, Sigma, St. Louis, MO, USA), and their maturity was determined according to the presence of a first polar body by microscope. In all cases, the mature oocytes were inseminated by ICSI. After ICSI, normal fertilization was confirmed when two distinct pronuclei were present 16-18 hours later. If the in vitro matured oocytes produced good quality cleaved embryos, they were transferred transcervically. Luteal support was provided by $4 \mathrm{mg}$ of estrogen valerate taken orally daily (Progynova, Schering-plough, Whitehouse Station, NJ, USA) beginning on the oocye retrieval day and $50 \mathrm{mg}$ progesterone in oil was also taken daily (Genefer progesterone, Watson Laboratories Inc., Corona, CA, USA) beginning on the day of ICSI up to gestational age 7 weeks.

2) In the GnRH agonist long protocol group (group B), pituitary suppression was initiated with $\mathrm{GnRH}$ agonist $(0.5 \mathrm{mg}$ buserelin acetate, Superfact, Sanofi-Aventis, Frankfurt, Germany or $0.1 \mathrm{mg}$ leuprolide acetate, Lucrin, Abbott Lab, Chicago, IL, USA) from the mid-luteal phase of the previous cycle to the day of $\mathrm{hCG}$ administration. On day 2 to 3 of the menstrual cycle, the pelvic cavity was examined and blood samples were obtained by venipuncture for assays of $F S H, E_{2}$, and $\mathrm{LH}$.

3) In the GnRH antagonist multi-dose flexible protocol (group C), 
stimulation using gonadotropin started on menstrual day 2 or 3, and GnRH antagonist (0.25 mg cetrorelix acetate, Cetrotide, Serono, Geneva, Switzerland or ganirelix acetate, Orgalutran, Schering-Plough) was administered when the follicles were more than $12 \mathrm{~mm}$ or serum $\mathrm{E}_{2}$ concentrations were greater than $200 \mathrm{pg} / \mathrm{mL}$.

The dosage of gonadotrophin was determined by the age of the patient and the ovarian response of the previous cycle. The stimulation was monitored by assessments of follicle size and serum $E_{2}$ levels in groups $B$ and $C$. In groups $B$ and $C$, when a leading follicle was $>18$ $\mathrm{mm}$ in diameter, hCG (250 $\mu \mathrm{g}$ of choriogonadotropin-alfa, Ovidrel) was administered. TVS-guided oocyte retrieval was performed 36 hours after the hCG injection. The embryos were transferred into the uterine cavity on day 3 after oocyte retrieval. Pregnancy was determined by a serial serum $\beta$-hCG level of $>5 \mathrm{mlL} / \mathrm{mL} 12$ days after the oocyte retrieval. Clinical pregnancy was defined as the presence of a gestational sac by ultrasonography at approximately 5 weeks of pregnancy.

\section{Statistical analysis}

All analyses were performed using SPSS ver. 12.0 (SPSS Inc., Chicago, IL, USA). One-way analysis of variance was used for statistical analysis. Each variable is presented as the mean \pm SD. A $p<0.05$ was considered statistically significant.

\section{Results}

Age, BMI, basal FSH and LH levels, and duration of infertility did not differ among the three groups (group A: 14 cycles, IVM/IVF with FSH and hCG priming protocol; group B: 14 cycles, GnRH agonist long protocol; group C: 39 cycles, GnRH antagonist multi-dose flexible protocol). The total doses of gonadotropin (group A: 1,008.3 \pm 326.3 vs. group $B: 2,700.0 \pm 824.3$ vs. group C: $1,657.7 \pm 669.8 \mathrm{IU} ; p=0.001$ ) and stimulation duration were significantly lower in group $A$ than groups B and C (group A: $5.2 \pm 0.9$ vs. group B: $11.6 \pm 2.0$ vs. group C: $10.6 \pm 2.4$ days; $p=0.00$, respectively). Endometrial thickness on the day hCG was administered was significantly lower in group $A$ than group B (Table 1).

The number of retrieved oocytes (group A: $9.9 \pm 5.1$ vs. group B: $27.6 \pm 10.7$ vs. group $C: 19.4 \pm 11.1 ; p=0.00)$ and the number of 2 pronucleus embryos (group A: $4.8 \pm 2.4$ vs. group $B: 16.4 \pm 6.5$ vs. group $C: 12.4 \pm 7.2 ; p=0.00$ ) were significantly lower in group $A$ than groups $B$ and $C$. In group $A$, the maturation rate was $63.1 \%$ and the fertilization rate was $75.5 \%$, which was similar to groups B (75.6\%) and C (77.8\%). Embryo transfer was cancelled in two cycles of group $B$ and in 10 cycles of group C. The implantation rate (group A: $24.4 \%$ vs. group B: $25.0 \%$ vs. group C: $23.3 \%$ ) did not differ among the three groups. The CPR (group A: $53.8 \%$ vs. group B: $42.9 \%$ vs. group C: $51.7 \%$ ), MR (group A: $14.3 \%$ vs. group B: $0 \%$ vs. group C: $10.3 \%$ ), and LBR (group A: $38.5 \%$ vs. group B: $41.7 \%$ vs. group C: $34.5 \%$ ) per embryo transfer did not differ among the three groups. In group B, 1 pregnancy had a 2nd trimester loss. In group C, 1 patient had an ectopic pregnancy. There was no incidence of OHSS in group $A$, and the differences from groups $B$ and $C$ were insignificant (group $A: 0 \%$ vs. group B: 14.3\% vs. group C: 17.9\%) (Table 2).

\section{Discussion}

This is the first study to compare IVM outcomes with either a GnRH agonist or $\mathrm{GnRH}$ antagonist cycle in infertile women with PCOS. Furthermore, this is the only prospective study that has attempted to compare implantation and live birth rates across IVM and conventional IVF cycles, with no significant differences being seen in either rate.

In general, implantation and live birth rates of IVM embryos are still lower than those of conventional IVF embryos [13]. These findings may be explained by the fact that embryos derived from IVM have

Table 1. Basal characteristics of the study population according to IVF protocol

\begin{tabular}{lccc}
\hline Characteristic & IVM (group A) & GnRH agonist (group B) & GnRH antagonist (group C) \\
\hline No. of patients & 11 & 14 & 36 \\
No. of cycles & 14 & 14 & 39 \\
Age $(\mathrm{yr})$ & $31.2 \pm 3.7$ & $34.4 \pm 3.8$ & $32.9 \pm 2.9$ \\
Duration of infertility $(\mathrm{mo})$ & $27.6 \pm 15.0$ & $59.8 \pm 42.8$ & $45.0 \pm 25.8$ \\
Body mass index $\left(\mathrm{kg} / \mathrm{m}^{2}\right)$ & $24.6 \pm 4.9$ & $23.6 \pm 4.0$ & $23.3 \pm 4.3$ \\
Basal FSH $(\mathrm{mlU} / \mathrm{mL})$ & $6.49 \pm 1.85$ & $7.09 \pm 2.32$ & $7.43 \pm 2.15$ \\
Basal LH $(\mathrm{mlU} / \mathrm{mL})$ & $3.62 \pm 2.39$ & $5.41 \pm 4.55$ & $4.66 \pm 3.09$ \\
Duration of stimulation $($ day) & $5.2 \pm 0.9^{\mathrm{a}, \mathrm{b}}$ & $11.6 \pm 2.0$ & $10.6 \pm 2.4$ \\
Total dose of gonadotropin $(\mathrm{IU})$ & $1,008.3 \pm 326.3^{\mathrm{a}}$ & $2,700.0 \pm 824.3$ & $1,656.7 \pm 669.77^{\mathrm{c}}$ \\
Endometrium thickness $(\mathrm{mm})$ & $9.2 \pm 1.5^{\mathrm{a}}$ & $11.8 \pm 2.3$ & $10.5 \pm 1.89$ \\
\hline
\end{tabular}

Values are expressed as the mean \pm SD. $p$-value $<0.05$, statistically significant.

${ }^{\mathrm{a} G r o u p ~ A ~ v s . ~ g r o u p ~ B, ~}{ }^{\mathrm{b}}$ group A vs. group C, 'group B vs. group C. 
Table 2. ART outcomes according to IVF protocol

\begin{tabular}{lccc}
\hline & IVM (group A) & GnRH-agonist (group B) & GnRH-antagonist (group C) \\
\hline No. of cycles & 14 & 14 & 39 \\
No. of retrieved oocytes & $9.9 \pm 5.1^{\mathrm{a}, \mathrm{b}}$ & $27.6 \pm 10.7$ & $19.4 \pm 11.1$ \\
No. of mature oocytes & $1.3 \pm 1.8^{\mathrm{a}, \mathrm{b}}$ & $19.9 \pm 8.5$ & $3.8 \pm 9.5$ \\
No. of immature oocytes & $7.7 \pm 5.3$ & $4.2 \pm 2.9$ & $7.0 \pm .4$ \\
Maturation rate (\%) & $63.1 \pm 31.2^{\mathrm{a}}$ & $73.9 \pm 17.9$ & $16.9 \pm 18.3$ \\
No. of pronucleus embryos & $4.8 \pm 2.4^{\mathrm{a}, \mathrm{b}}$ & $16.4 \pm 6.5$ & $7.4 \pm 7.2$ \\
Fertilization rate (\%) & $75.0 \pm 25.6$ & $75.6 \pm 14.5$ & $2.5 \pm 17.4$ \\
No. of transferred embryos & $3.2 \pm 0.6$ & $3.3 \pm 0.5$ & $4.0 \pm 2.2$ \\
No. of good quality embryos & $2.2 \pm 2.3$ & $4.3 \pm 2.9$ & $15 / 39(38.5)$ \\
CPR/attempt (\%) & $7 / 14(50)$ & $6 / 14(42.9)$ & $15 / 29(51.7)$ \\
CPR/ET (\%) & $7 / 13(53.8)$ & $6 / 12(50)$ & $4 / 39(10.3)$ \\
MR (\%) & $2 / 14(14.3)$ & $0 / 14(0)$ & $17 / 73(23.3)$ \\
IR (\%) & $10 / 41(24.4)$ & $10 / 40(25)$ & $10 / 39(25.6)$ \\
LBR/attempt (\%) & $5 / 14(35.7)$ & $5 / 14(35.7)$ & $10 / 29(34.5)$ \\
LBR/ET (\%) & $5 / 13(38.5)$ & $5 / 12(41.7)$ & $7 / 39(17.9)$ \\
No. of OHSS (\%) & $0 / 14(0)$ & $2 / 14(14.3)$ & \\
After singleton pregnancy & & & \\
hCG level on 12 day after OPU (mlU/mL) & $79.6 \pm 64.3$ & $129.8 \pm 28.3$ & $149.2 \pm 116.1$ \\
hCG level on 14 day after OPU (mlU/mL) & $173.5 \pm 156.8$ & \\
\hline
\end{tabular}

Values are presented as mean $\pm \mathrm{SD}$ or number (\%). $p$-value $<0.05$, tatistically significant.

ART, assisted reproductive technology; CPR, clinical pregnancy rate; ET, embryo transfer; MR, miscarriage rate; IR, implantation rate; LBR, live birth rate; OHSS, ovarian hyperstimulation syndrome; OPU, oocyte pick-up.

aGroup A vs. group B, broup A vs. group C, 'group B vs. group C.

lower implantation potential than those derived from conventional IVF. As aneuploidy is a major cause of embryonic and fetal loss, it may be postulated that the higher incidence of chromosomal abnormalities in embryos derived from IVM oocytes may be a factor limiting the implantation potential of IVM embryos. Requena et al. [14] reported a higher aneuploidy rate (60\%) in IVM embryos than in a control group (33\%), in women with the risk of sex chromosome-linked diseases, based on FISH analysis of chromosomes 13, 15, 16, 18, 21, $22, X$, and $Y$. It may be hypothesized that the higher incidence of chromosomal abnormalities, together with other cytoplasmic alterations due to the altered maturation of organelles in IVM, may account for the early cleavage arrest and low implantation rate. In the present study, we demonstrated an implantation rate of $24.4 \%$ with the FSH and hCG priming IVM protocol, which is comparable to that of conventional IVF cycles.

Although there have been conflicting reports on the clinical outcomes of FSH or hCG priming before IVM, advantages of early follicular phase FSH or hCG priming IVM cycles have been reported [10,11]. FSH priming in the early follicular phase may enhance follicular development and the maturational competence of immature oocytes. FSH receptors are exclusive to the granulosa cells of maturing follicles $[15,16]$, which in turn are directly connected to the oocyte via gap junctions $[17,18]$. FSH effects on cumulus cells may therefore be directly transmitted to the oocyte via secondary messenger CAMP and one of its downstream target kinases [19,20]. Roberts et al. [21], using a mouse model, found that FSH accelerates oocyte maturation in COC undergoing IVM.

The application of hCG priming before immature oocyte retrieval has been an established IVM protocol since the first report of improved pregnancy rates after hCG priming $[22,23]$. In the present study, oocyte retrieval was performed 38 hours after $\mathrm{hCG}$ injection. The extension of the interval between $\mathrm{hCG}$ priming and oocyte retrieval to 38 hours promoted IVM, and prolonged hCG stimulation was positively correlated with the number of oocytes that matured in vitro [12].

When embryos were categorized based on the maturation time of the oocytes in IVM cycles, embryos derived from oocytes that matured 48 hours after retrieval had higher rates of chromosomal abnormalities than embryos derived from oocytes that matured in the first 24 hours after retrieval [24]. Possible interactions between FSH and hCG priming with immature oocyte retrieval 38 hours later and accelerated IVM may decrease the aneuploidy rate. In the present study, embryos derived from 24 hour mature oocytes may be preferentially transferred, which may increase the implantation potential as the aneuploidy rate is decreased with earlier IVM.

In IVM cycles, to overcome the lower implantation rate, another possible strategy is to transfer greater numbers of embryos derived from IVM. In the present study, we detected no differences in the 
mean numbers of transferred embryos between IVM and conventional IVF cycles. The initial $\beta$-hCG levels in successful IVM cycles were higher than either the $\mathrm{GnRH}$ agonist or $\mathrm{GnRH}$ antagonist cycles. It is likely that the higher $\beta$-hCG levels in the IVM group are responsible for the increased implantation potential, as there were no differences in the number of transferred embryos, as an initial high serum $\beta$-hCG level generally correlates favorably with pregnancy outcome.

In addition, the clinical abortion rate and live birth rate were not significantly different among the three groups in the present study. There are few reports of the clinical abortion rate in previous IVM studies, which ranged from $9 \%$ to $37 \%$ [25-27]. The clinical abortion rate after IVM tends to be higher than that of conventional IVF cycles. However, the reasons for the increased risk of clinical abortion after IVM remain unclear. In the present study, the clinical abortion rate in the IVM cycles was $14.3 \%$, which was lower than the $25.3 \%$ seen in the largest study to address clinical abortion rates in 99 IVM cycles [28]. Although our sample was too small for a precise comparison, the lower clinical abortion rate we observed may be explained by reduced aneuploidy in the transferred embryos using the IVM protocol, with FSH and hCG priming with oocyte retrieval 38 hours later. The increased implantation potential with decreased clinical abortion with the FSH and hCG priming IVM protocol may have contributed to the live birth rates of $38.8 \%$, which is comparable to either of the conventional IVF cycle types.

IVM provides the benefits of avoiding risks associated with OHSS, and there was no incidence of OHSS in this study. There was no difference in OHSS incidence between the GnRH agonist and GnRH antagonist cycles. A recent meta-analysis showed no significant differences in the incidence of severe OHSS with GnRH antagonist cycles as compared with $\mathrm{GnRH}$ agonist cycles [29]. A potential limitation associated with the present study are phenotypic differences between the infertile women with PCOS included in the sample, despite satisfying the Rotterdam criteria for inclusion in this study. In particular, women with PCOS who are at higher risk of OHSS may elect for IVM in place of conventional IVF.

In conclusion, in this study, the implantation and live birth rate in IVM were comparable to those of GnRH agonist or GnRH antagonist cycles. Although our sample is too small to make definitive conclusions, the present IVM protocol, FSH and hCG priming with immature oocyte retrieval 38 hours later, is an effective ART option comparable to conventional IVF for infertile women with PCOS. A large scale prospective study is warranted.

\section{References}

1. Knochenhauer ES, Key TJ, Kahsar-Miller M, Waggoner W, Boots $L R$, Azziz R. Prevalence of the polycystic ovary syndrome in un- selected black and white women of the southeastern United States: a prospective study. J Clin Endocrinol Metab 1998;83: 3078-82.

2. Diamanti-Kandarakis E, Kouli C, Tsianateli T, Bergiele A. Therapeutic effects of metformin on insulin resistance and hyperandrogenism in polycystic ovary syndrome. Eur J Endocrinol 1998; 138:269-74.

3. Rotterdam ESHRE/ASRM-Sponsored PCOS Consensus Workshop Group. Revised 2003 consensus on diagnostic criteria and longterm health risks related to polycystic ovary syndrome. Fertil Steril 2004;81:19-25.

4. Thessaloniki ESHRE/ASRM-Sponsored PCOS Consensus Workshop Group. Consensus on infertility treatment related to polycystic ovary syndrome. Fertil Steril 2008;89:505-22.

5. MacDougall MJ, Tan SL, Balen A, Jacobs HS. A controlled study comparing patients with and without polycystic ovaries undergoing in-vitro fertilization. Hum Reprod 1993;8:233-7.

6. Griesinger G, Diedrich K, Tarlatzis BC, Kolibianakis EM. GnRH-antagonists in ovarian stimulation for IVF in patients with poor response to gonadotrophins, polycystic ovary syndrome, and risk of ovarian hyperstimulation: a meta-analysis. Reprod Biomed Online 2006;13:628-38.

7. Cha KY, Koo JJ, Ko JJ, Choi DH, Han SY, Yoon TK. Pregnancy after in vitro fertilization of human follicular oocytes collected from nonstimulated cycles, their culture in vitro and their transfer in a donor oocyte program. Fertil Steril 1991;55:109-13.

8. Buckett WM, Chian RC, Holzer H, Dean N, Usher R, Tan SL. Obstetric outcomes and congenital abnormalities after in vitro maturation, in vitro fertilization, and intracytoplasmic sperm injection. Obstet Gynecol 2007;110:885-91.

9. Siristatidis CS, Maheshwari A, Bhattacharya S. In vitro maturation in sub fertile women with polycystic ovarian syndrome undergoing assisted reproduction. Cochrane Database Syst Rev 2009; (1):CD006606.

10. Son WY, Yoon SH, Lim JH. Effect of gonadotrophin priming on invitro maturation of oocytes collected from women at risk of OHSS. Reprod Biomed Online 2006;13:340-8.

11. Son WY, Chung JT, Demirtas E, Holzer H, Sylvestre C, Buckett W, et al. Comparison of in-vitro maturation cycles with and without in-vivo matured oocytes retrieved. Reprod Biomed Online 2008; 17:59-67.

12. Son WY, Chung JT, Chian RC, Herrero B, Demirtas E, Elizur S, et al. A $38 \mathrm{~h}$ interval between $\mathrm{hCG}$ priming and oocyte retrieval increases in vivo and in vitro oocyte maturation rate in programmed IVM cycles. Hum Reprod 2008;23:2010-6.

13. Son WY, Chung JT, Herrero B, Dean N, Demirtas E, Holzer H, et al. Selection of the optimal day for oocyte retrieval based on the di- 
ameter of the dominant follicle in hCG-primed in vitro maturation cycles. Hum Reprod 2008;23:2680-5.

14. Requena A, Bronet F, Guillén A, Agudo D, Bou C, García-Velasco $J A$. The impact of in-vitro maturation of oocytes on aneuploidy rate. Reprod Biomed Online 2009;18:777-83.

15. Camp TA, Rahal JO, Mayo KE. Cellular localization and hormonal regulation of follicle-stimulating hormone and luteinizing hormone receptor messenger RNAs in the rat ovary. Mol Endocrinol 1991;5:1405-17.

16. O'Shaughnessy PJ, Dudley K, Rajapaksha WR. Expression of follicle stimulating hormone-receptor mRNA during gonadal development. Mol Cell Endocrinol 1996;125:169-75.

17. Albertini DF, Anderson E. The appearance and structure of intercellular connections during the ontogeny of the rabbit ovarian follicle with particular reference to gap junctions. J Cell Biol 1974; 63:234-50.

18. Anderson E, Albertini DF. Gap junctions between the oocyte and companion follicle cells in the mammalian ovary. J Cell Biol 1976; 71:680-6.

19. Richards JS. New signaling pathways for hormones and cyclic adenosine 3',5'-monophosphate action in endocrine cells. Mol Endocrinol 2001;15:209-18.

20. Gonzalez-Robayna IJ, Falender AE, Ochsner S, Firestone GL, Richards JS. Follicle-Stimulating hormone (FSH) stimulates phosphorylation and activation of protein kinase $B$ (PKB/Akt) and serum and glucocorticoid-Induced kinase (Sgk): evidence for A kinaseindependent signaling by FSH in granulosa cells. Mol Endocrinol 2000;14:1283-300.

21. Roberts R, Stark J, latropoulou A, Becker DL, Franks S, Hardy K. Energy substrate metabolism of mouse cumulus-oocyte complexes: response to follicle-stimulating hormone is mediated by the phosphatidylinositol 3-kinase pathway and is associated with oocyte maturation. Biol Reprod 2004;71:199-209.

22. Chian RC, Gülekli B, Buckett WM, Tan SL. Priming with human chorionic gonadotropin before retrieval of immature oocytes in women with infertility due to the polycystic ovary syndrome. N Engl J Med 1999;341:1624-6.

23. Chian RC, Buckett WM, Tulandi T, Tan SL. Prospective randomized study of human chorionic gonadotrophin priming before immature oocyte retrieval from unstimulated women with polycystic ovarian syndrome. Hum Reprod 2000;15:165-70.

24. Zhang XY, Ata B, Son WY, Buckett WM, Tan SL, Ao A. Chromosome abnormality rates in human embryos obtained from in-vitro maturation and IVF treatment cycles. Reprod Biomed Online 2010;21:552-9.

25. Cha KY, Chung HM, Lee DR, Kwon H, Chung MK, Park LS, et al. Obstetric outcome of patients with polycystic ovary syndrome treated by in vitro maturation and in vitro fertilization-embryo transfer. Fertil Steril 2005;83:1461-5.

26. Le Du A, Kadoch IJ, Bourcigaux N, Doumerc S, Bourrier MC, Chevalier $\mathrm{N}$, et al. In vitro oocyte maturation for the treatment of infertility associated with polycystic ovarian syndrome: the French experience. Hum Reprod 2005;20:420-4.

27. Lin $\mathrm{YH}$, Hwang JL. In vitro maturation of human oocytes. Taiwan J Obstet Gynecol 2006;45:95-9.

28. Buckett WM, Chian RC, Dean NL, Sylvestre C, Holzer HE, Tan SL. Pregnancy loss in pregnancies conceived after in vitro oocyte maturation, conventional in vitro fertilization, and intracytoplasmic sperm injection. Fertil Steril 2008;90:546-50.

29. Pundir J, Sunkara SK, El-Toukhy T, Khalaf Y. Meta-analysis of GnRH antagonist protocols: do they reduce the risk of OHSS in PCOS? Reprod Biomed Online 2012;24:6-22. 